\author{
EUROPEAN ORGANIZATION FOR NUCLEAR RESEARCH \\ CERN - SL DIVISION
}

CERN-SL-2000-054 BI

\title{
PERFORMANCE OF BPM ELECTRONICS FOR THE LEP SPECTROMETER
}

\author{
Barbero E*., Bergoz $\mathbf{J}^{+}$., Dehning B*., Matheson J*., Prochnow \\ $\mathbf{J}^{\dagger}$., Torrence $\mathbf{E}^{*}$., Unser $\mathbf{K}^{+}$. and Vismara $\mathbf{G}^{*}$. \\ * CERN, Geneva, Switzerland \\ ${ }^{\dagger}$ RWTH, Aachen, Germany \\ + Bergoz Instrumentation, 01630 St. Genis Pouilly, France
}

\begin{abstract}
At the LEP e+/e- collider at CERN, Geneva, a Spectrometer is used to determine the beam energy with a relative accuracy of $10^{-4}$.

The Spectrometer measures the change in bending angle in a well-characterised dipole magnet as LEP is ramped. The beam trajectory is obtained using three beam position monitors (BPMs) on each side of the magnet. The error on each BPM measurement should not exceed 1 micron if the desired accuracy on the bending angle is to be reached.

The BPMs used consist of an aluminium block with an elliptical aperture and four capacitive button pickup electrodes. The button signals are fed to customised electronics supplied by Bergoz. The electronics use time multiplexing of individual button signals through a single processing chain to optimise for longterm stability.

We report on our experience of the performance of these electronics, describing measurements made with test signals and with beam. We have implemented a beam-based calibration procedure and have monitored the reproducibility of the measurements obtained over time. Measurements show that a relative accuracy better than $300 \mathrm{~nm}$ is achievable over a period of $1 \mathrm{hr}$.
\end{abstract}




\title{
Performance of BPM Electronics for the LEP Spectrometer
}

\author{
E.Barbero*, J.Bergoz ${ }^{+}$, B.Dehning*, J.Matheson*, \\ J.Prochnow $^{\dagger}$, E.Torrence*, K.Unser ${ }^{+}$G.Vismara* \\ ${ }^{*}$ CERN, Geneva, Switzerland ${ }^{\dagger} R W T H$, Aachen, Germany \\ ${ }^{+}$Bergoz Instrumentation, 01630 St. Genis Pouilly, France
}

\begin{abstract}
At the LEP e+/e- collider at CERN, Geneva, a Spectrometer is used to determine the beam energy with a relative accuracy of $10^{-4}$.

The Spectrometer measures the change in bending angle in a well-characterised dipole magnet as LEP is ramped. The beam trajectory is obtained using three beam position monitors (BPMs) on each side of the magnet. The error on each BPM measurement should not exceed 1 micron if the desired accuracy on the bending angle is to be reached.

The BPMs used consist of an aluminium block with an elliptical aperture and four capacitive button pickup electrodes. The button signals are fed to customised electronics supplied by Bergoz. The electronics use time multiplexing of indidvidual button signals through a single processing chain to optimise for long-term stability.

We report on our experience of the performance of these electronics, describing measurements made with test signals and with beam. We have implemented a beam-based calibration procedure and have monitored the reproducibilty of the measurements obtained over time. Measurements show that a relative accuracy better than $300 \mathrm{~nm}$ is achievable over a period of $1 \mathrm{hr}$.
\end{abstract}

\section{INTRODUCTION}

In the year 2000 running, the LEP machine at CERN will be used to study decays of $\mathrm{W}$ bosons at energies in excess of $100 \mathrm{GeV}$ per beam. In order to reconstruct the $\mathrm{W}$ mass with high precision, the beam energy calibration should have an uncertainty better than $15 \mathrm{MeV}$ per beam. Resonant spin depolarisation (RDP) remains the most accurate procedure for the calibraton of beam energy [1]; however, above $60 \mathrm{GeV}$, a sufficiently high polarisation level cannot be achieved. At physics energies, the beam energy is calculated from the bending magnetic field of LEP using nuclear magnetic resonance (NMR) probes [2]. The limited accuracy of the NMR-based measurement resulted in a proposal to build the LEP Spectrometer [3] as an alternative method of energy determination. The Spectrometer consists of a steel dipole magnet equipped with reference NMR probes. The integral field of 


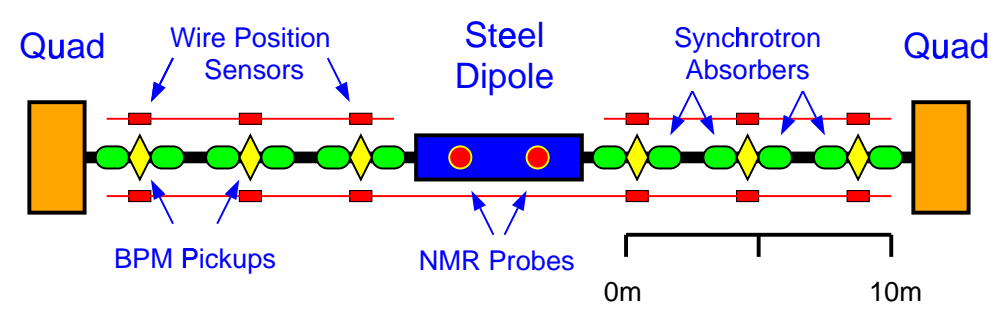

FIGURE 1. Layout of the LEP Spectrometer

the magnet has been measured as a function of the reference probe readings with a relative accuracy of $3 \times 10^{-5}$ [4]. At each side of the magnet, there are three BPM stations for the reconstruction of the beam bending angle (Fig. 1). As LEP is ramped from the injection energy to the physics energy, the power radiated as synchrotron light rises from zero to $\sim 1 \mathrm{~kW} / \mathrm{m}$. In order to prevent motion due to thermal expansion, the magnet and BPMs are equipped with cooling stations which allow more accurate control than is possible with the standard LEP system. Any remaining thermally-induced motion can be detected using a stretched-wire positioning system and corrected for in the subsequent data analysis. The BPM buttons themselves are shielded from the synchrotron radiation by copper absorbers.

The Spectrometer is calibrated at low energy against resonant depolarisation, avoiding the need for an absolute angle measurement. The target accuracy is $\delta E / E<1 \times 10^{-4}$, which sets a limit on the tolerable BPM error of $1 \mu \mathrm{m}$. This error corresponds to a relative electric field change of $2.5 \times 10^{-5}$ at the buttons. Nonlinearities in the response of the BPMs [5] necessitate the steering of the beam to the nominal trajectory before and after the ramp.

The pickup electronics were custom manufactured for the Spectrometer application, being based on a design for a synchrotron light source [6], with a specification of $\sim 1 \mu \mathrm{m}$ relative accuracy [7]. The electronics use the principle of time multiplexing of the four button signals through a common amplifier chain, switching at $400 \mathrm{~Hz}$. The LEP bunch frequency is $44.982017 \mathrm{kHz}$ and a bandpass filter with a width of $3.8 \mathrm{kHz}$ has been used to select the 1000 th harmonic at $44.98202 \mathrm{MHz}$. At the position of the spectrometer, the arrival time of the positron bunches precedes that of the electron bunches by $2.7 \mu \mathrm{s}$ and a gate is incorporated in the electronics to allow the selection of either or both beams.

As well as the $x$ and $y$ signals, the individual button signals are output from the cards, as well as the sum signal of all four buttons. The electronics uses automatic gain control (AGC) to keep the sum constant, independent of beam current and the AGC signal is also available at the output connector. 


\section{LABORATORY TESTS OF THE ELECTRONICS}

The BPM electronics cards were tested by using an input signal similar to that measured from the buttons in the presence of circulating beams. This signal was provided by a simulator card feeding four coaxial cables. The cables were connected in parallel at the simulator card to ensure the button inputs on the BPM electronics card received the same signal. Trigger pulses were derived from a R.F. generator running at $352.20971 \mathrm{MHz}$, through a divider to give $44.98202 \mathrm{kHz}$ at the simulator card. Between trigger pulses, a capacitor charges up from a high voltage supply; a trigger pulse then switches on an avalanche transistor and the discharge of the capacitor generates a signal at the cables. The pulse width was measured to be $\sim 1.3 \mathrm{~ns}$, with a jitter less than $\pm 0.3 \mathrm{ps}$, with an amplitude of $6.7 \mathrm{~V}$. Three simulator cards were built, allowing simultaneous testing of the BPM cards for one Spectrometer arm.

The simulated beam setup was used in the laboratory and also in the tunnel during the 1999 LEP shutdown period. In the latter situation, the simulator cards were situated in the LEP tunnel feeding the BPM cables from the front end. The BPM electronics cards were mounted in a rack, $200 \mathrm{~m}$ away in the pit at LEP Point 3.

\section{The Effect of Temperature Changes}
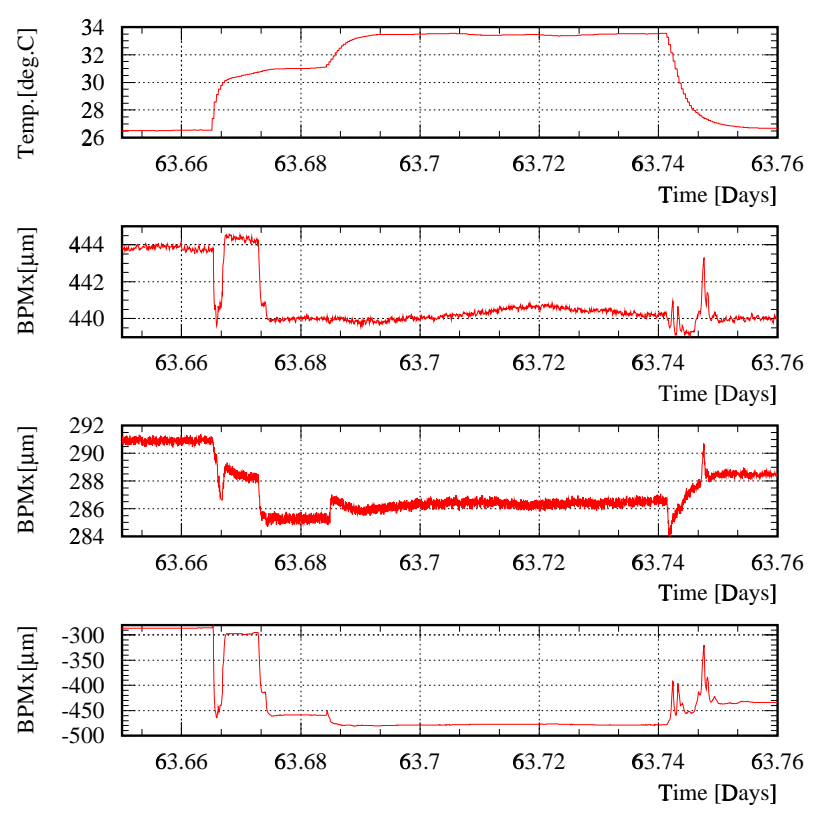

FIGURE 2. Effect of rack temperature on BPM x signals

In Figs. 2 and 3 variations in the BPM card temperatures were deliberately introduced by blowing warm air into the electronics rack. The figures show that 

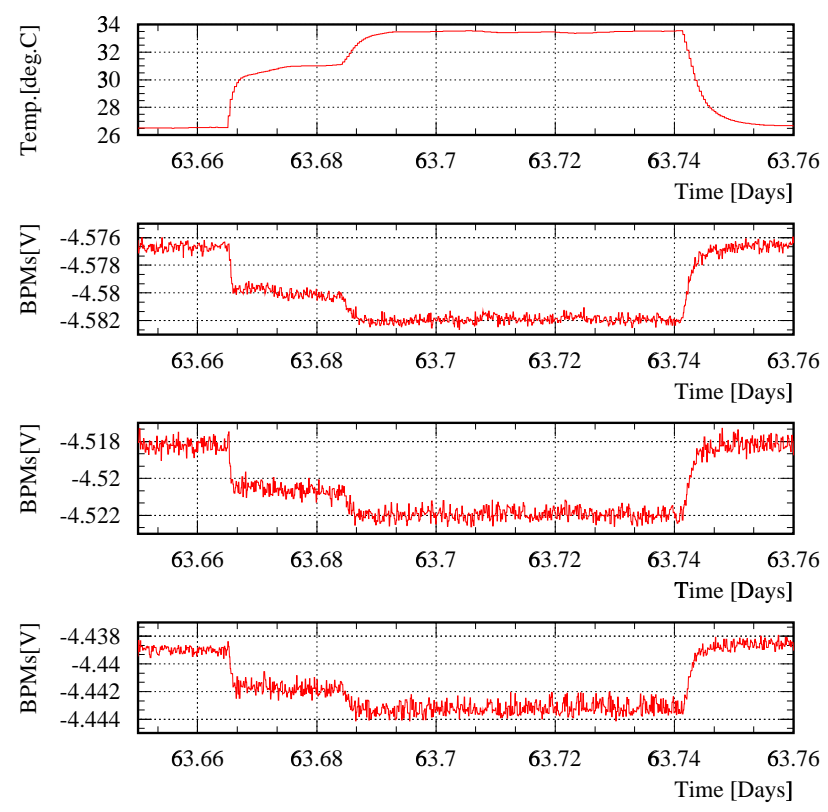

FIGURE 3. Effect of rack temperature on BPM sum signals

the temperature change with time leads to changes in the BPM position readings because the sum signal, which sets the automatic gain control, is changing. For the LEP 2000 running, the BPM electronics will be housed in a temperature-controlled rack to suppress such effects.

\section{The Effect of Signal Frequency Changes}

The effect of signal frequency changes on the BPM cards is shown in Fig. 4. The AGC and position signals show some dependence on signal frequency; however, the variation in frequency during normal LEP running should be less than $200 \mathrm{~Hz}$ so that no significant effect on the BPM performance is expected.

\section{The Effect of Beam Current Changes}

The effect of beam current changes was examined in the laboratory using a switchable attenuator unit. The four outputs of the simulator card were combined, fed through the attenuator and re-split into the four button inputs on the BPM card under test. Thus, the same attenuation was applied to each input channel. The results from this procedure are shown in Fig. 5, where the changing input signal results in the expected change in the AGC output (Fig. 5,top). 

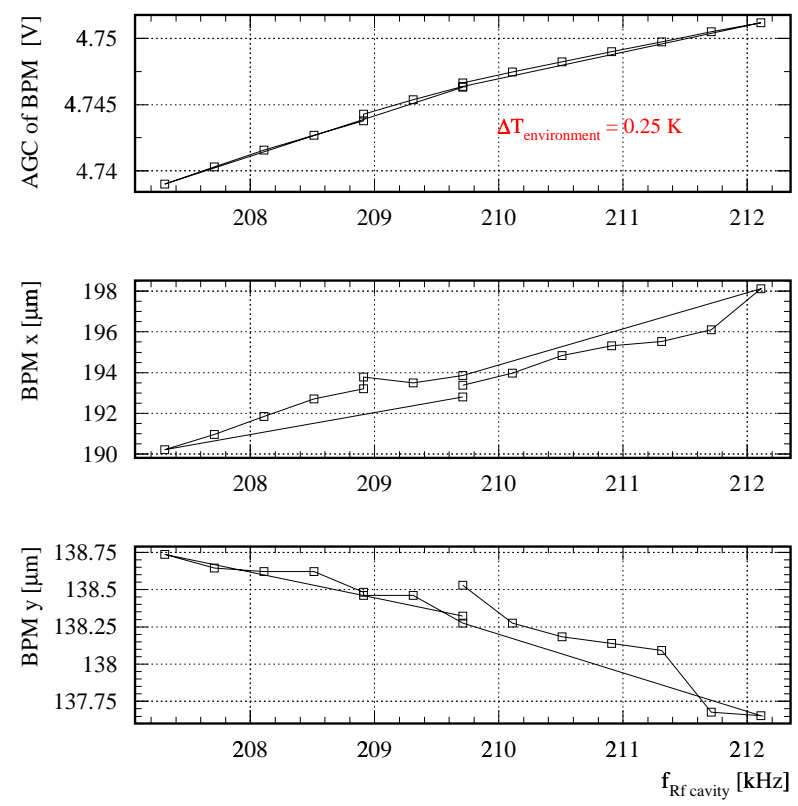

FIGURE 4. Effect of frequency on BPM card response
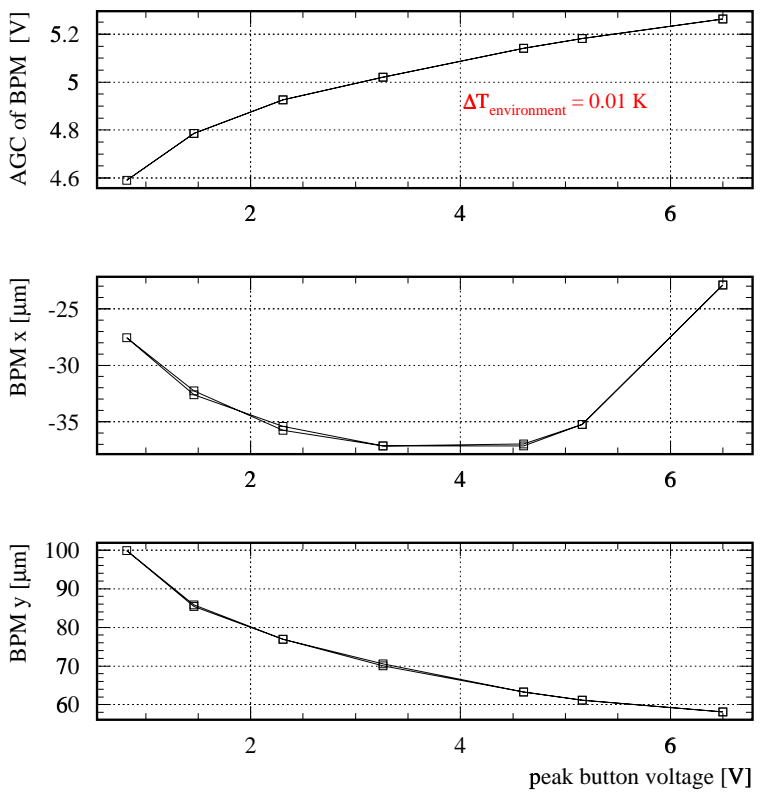

FIGURE 5. Effect of beam current with zero $\mathrm{x}$ offset 


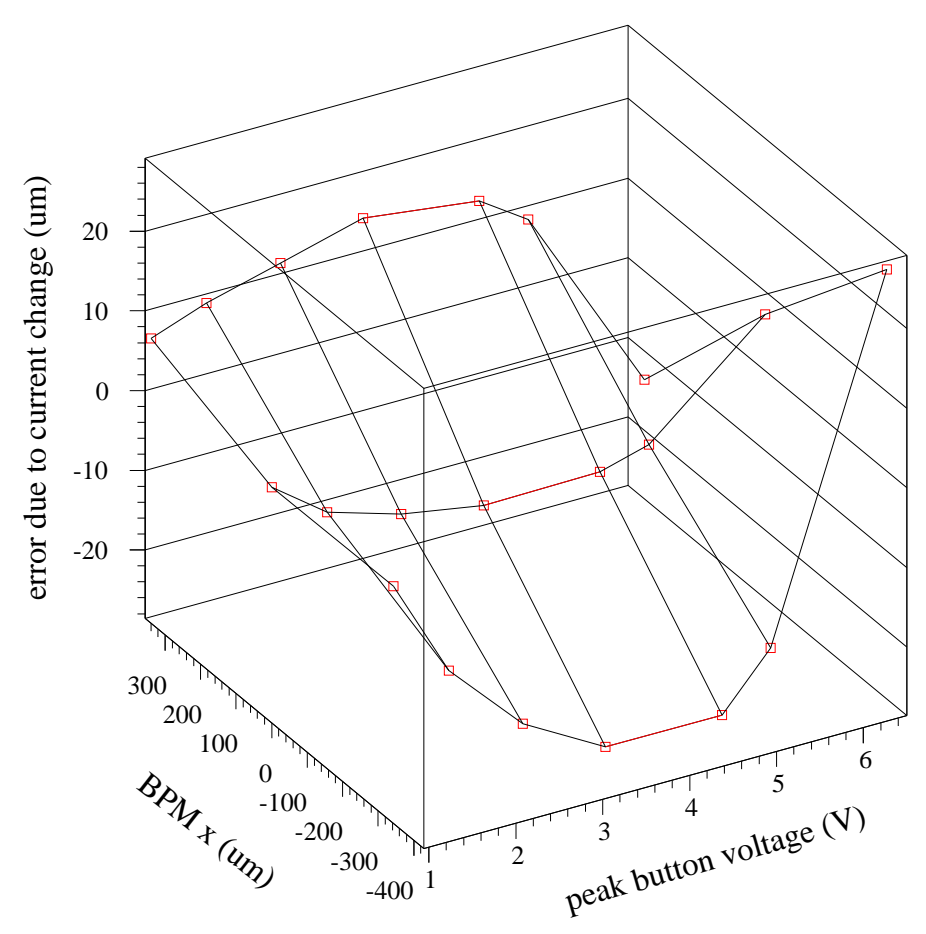

FIGURE 6. Effect of beam current with varying $\mathrm{x}$ offset

The $x$ and $y$ readings from the cards were found also to depend on beam current. This point was investigated further by adding two additional lengths of cable to attenuate the signal on two button inputs. The additional delay (10 ns) so introduced is not important compared to the integration time of the signal, which is of the order of ms. By the choice of buttons, a beam with a negative or positive offset of $\sim 350 \mu \mathrm{m}$ was simulated (Fig. 6); it was found that increasing beam offset tended to increase the beam current dependence of the position signal.

The dependence of the position signals on beam current is larger than expected, so that it is intended to minimise it using the on-card attenuators. In this way, the working point in the horizontal plane of the BPM electronics will be set in the range of 3.3-4.6 $\mathrm{V}$ at the input, allowing a beam current variation of $30 \%$, whilst keeping the resulting position changes below $1 \mu \mathrm{m}$.

\section{Stability of the beam position measurement}

With the simulator cards in the LEP tunnel and the BPM electronics in the pit at LEP point 3, their temperatures were reasonably independent. The temperature of the electronics rack in the pit was monitored continuously and its effect on the BPM readings observed. Fig. 7 shows the signals from one BPM card over a period of $7 \mathrm{hr}$ during which the temperature variation was less than $0.02 \mathrm{~K}$. Fig. 8 shows histograms of the position measurements accumulated over the same time 

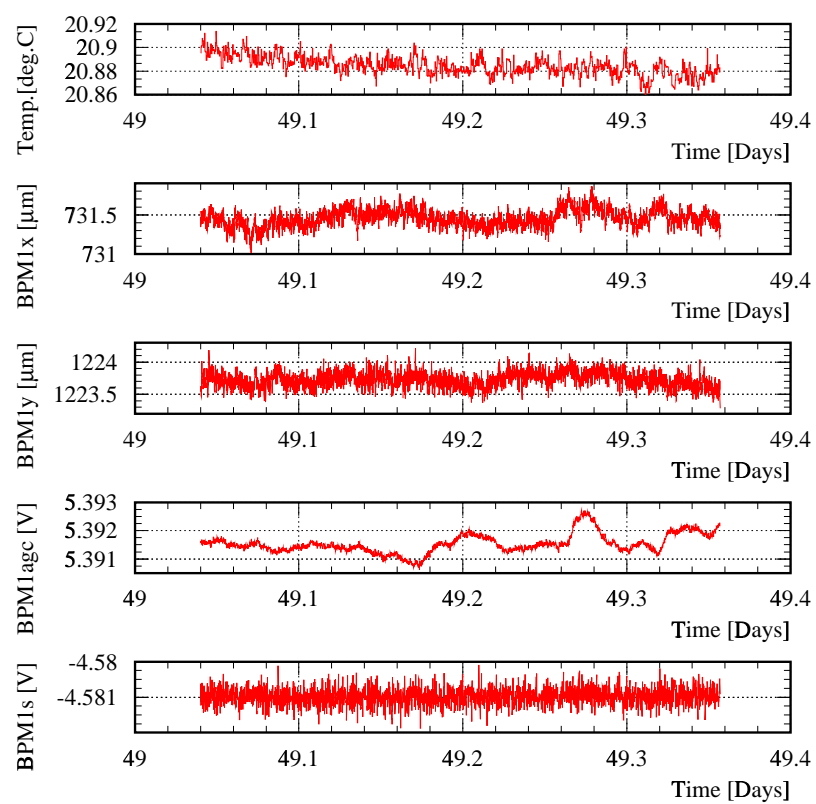

FIGURE 7. BPM1 signals vs time at constant temperature
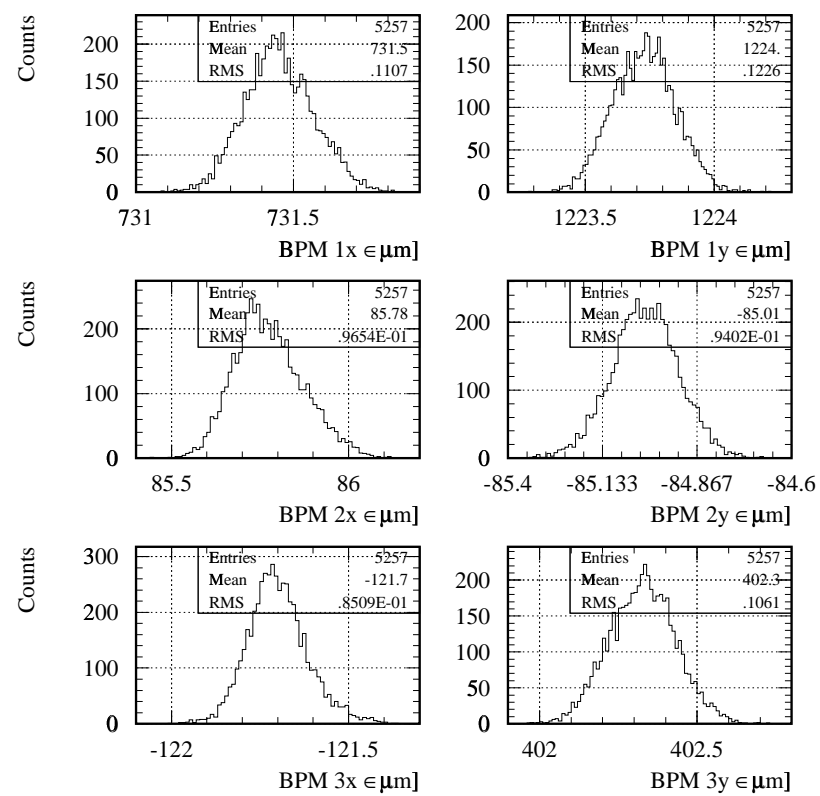

FIGURE 8. BPM position signal histograms at constant temperature 
period which demonstrate that, in the absence of temperature changes in BPM card environment, the BPM readings show variation with a $\sigma$ of only $100 \mathrm{~nm}$.

\section{BEAM TESTS OF THE ELECTRONICS}

\section{Cross-Calibration of the BPMs}
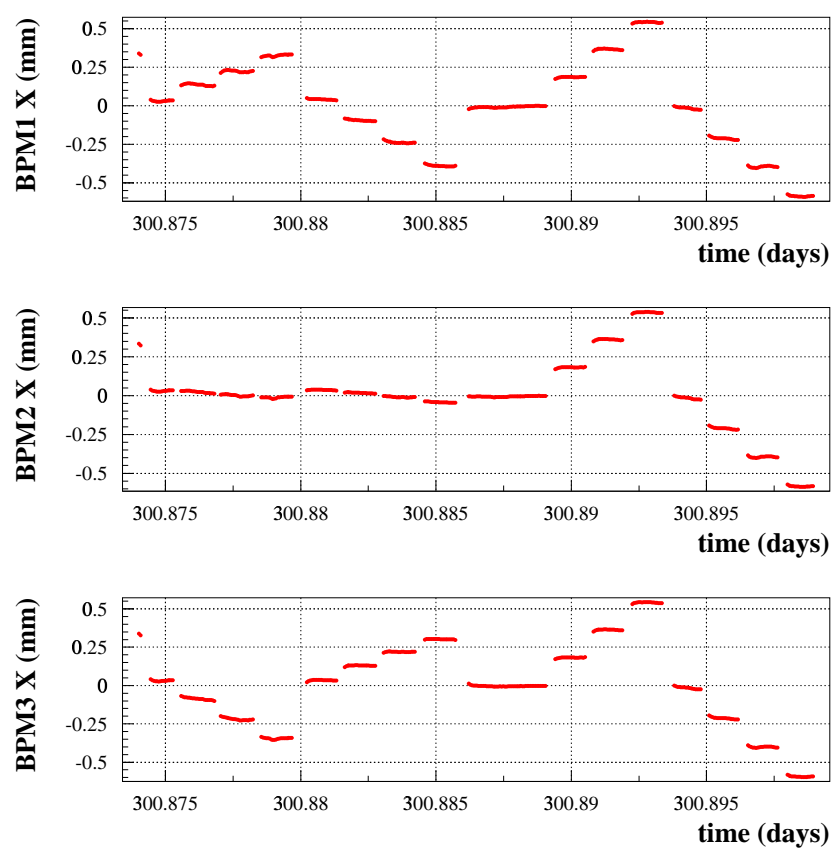

FIGURE 9. Bumps for cross-calibration of BPMs

The BPM electronics cards were supplied with a calibration to the level of a few \%. This absolute error on the calibration of the cards need not introduce a large error on the angle measured at the Spectrometer, as long as the relative calibration is adequate. For this reason, a beam-based cross-calibration technique was devised.

The beam movements used are shown in Fig. 9, which refers to one arm of the Spectrometer. A series of rotations of the beam is performed about the central $\mathrm{BPM}$, followed by a series of parallel orbit bumps, up to $\pm 600 \mu \mathrm{m}$. The aim of the procedure is to find the calibration of BPM 1 and BPM 3, in terms of that of BPM 2. In Fig. 10 (top left), the reading of BPM 2 is plotted against the readings of the other two, after subtraction of the mean value of each BPM data set. The two sets of beam movements result in two lines which together define a plane, the angles of which relative to the ideal plane $z-(x+y) / 2=0$ give the relative gains of the BPMs.

It is instructive to examine the triple residual for the BPM data; this is defined here as $R_{X}=X_{B P M 2}-\left(X_{B P M 1}+X_{B P M 3}\right) / 2 . \quad R_{X}$ would be zero for perfect 

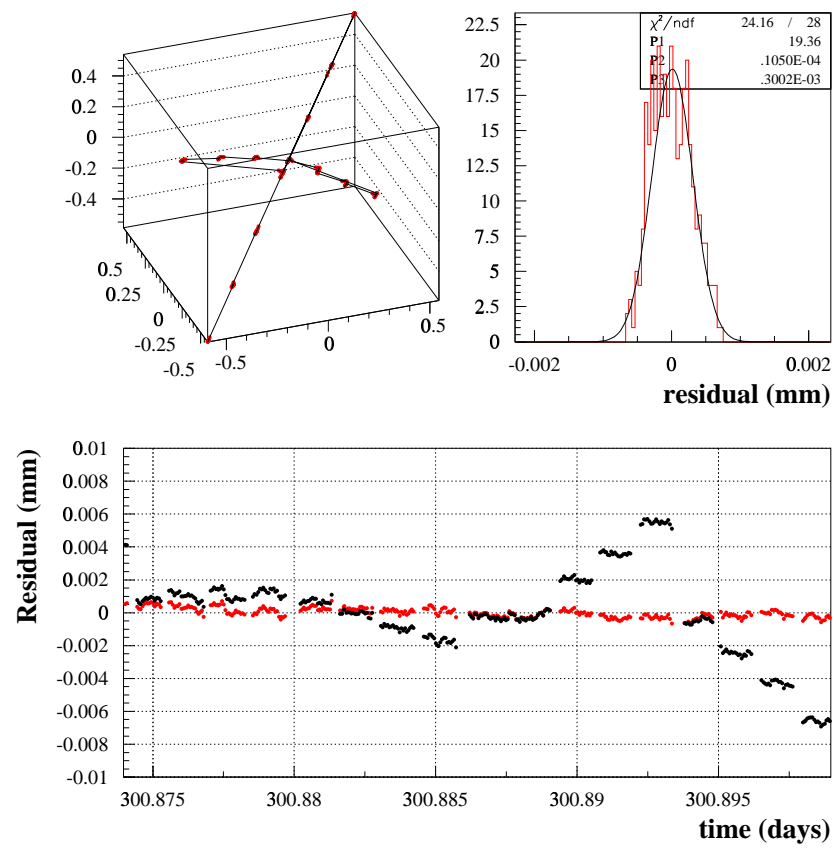

FIGURE 10. Results from cross-calibration of BPMs

BPMs, but in reality has a finite value due to noise and inaccuracies in the gain cross-calibration. Fig. 10 (bottom) also shows $R_{X}$ against time before and after correction of the gains to that of BPM 2. The histogram of $R_{X}$ has a $\sigma$ of $300 \mathrm{~nm}$ after the correction, over the time of 60 min taken to complete the sequence of beam movements. This implies that our requirement for sub- $\mu \mathrm{m}$ relative accuracy has been achieved, the fluctuations from one BPM being around $200 \mathrm{~nm}$. The crosscalibration before and after the ramp to high energy was found to give gain values which agreed to better than $0.25 \%$.

\section{CONCLUSIONS}

The BPM electronics for the LEP spectrometer have been tested using an electronically simulated beam pulse in LEP Point 3 itself and in the laboratory. We have investigated the effects of temperature, bunch frequency and beam current. The variation of bunch frequency required to cause a significant perturbation of the position measurement was far greater than that which will occur during normal LEP running. However, some dependence of the position measurement on beam current and some dependence on BPM card temperature was found. These effects will be minimised during running; the former by using the on-card attenuators to select the flattest part of the position versus current curve and the latter by housing the BPMs in a temperature-controlled rack. 
The relative calibration of the BPMs in the Spectrometer must be known sufficiently well to prevent systematic errors. We have described a beam-based calibration technique which results in the triple residual for each Spectrometer arm being comfortably less than $1 \mu \mathrm{m}$. Both beam-based and electronic measurements indicate that the BPM cards are sufficiently stable with time for the Spectrometer application.

\section{REFERENCES}

1. L.Arnaudon et al., Accurate determination of the LEP beam energy by resonant depolarization, Z. Phys. C66, 45-62 (1995)

2. A.Blondel et al., Evaluation of the LEP centre-of-mass energy above the $W$-pair production threshold, CERN-SL/98-073 (1998)

3. See for example M.Placidi in: Proceedings of the 8th LEP Performance Workshop, Chamonix CERN-SL-98-006-DI (1998)

4. F.Roncarolo, Diploma Thesis, Politecnico di Milano (2000)

5. J.Matheson, Nonlinearities in the Response of BPMs for the LEP Spectrometer, to be published

6. J.Hinkson, Advanced Light Source Beam Position Monitor, AIP Conference Proceedings No.252, Accelerator Instrumentation Workshop, p.21 (1991)

7. Bergoz Instrumentation, 01630 St. Genis Pouilly, France 